\title{
The taste of scales and chords
}

\author{
Alan Taitz ${ }^{1}$, Diego Shalom ${ }^{1}$, Marcos A Trevisan ${ }^{1}$ and Bruno Mesz ${ }^{2}$
}

\begin{abstract}
${ }^{1}$ Dept. of Physics, University of Buenos Aires and Institute of Physics Buenos Aires (IFIBA) CONICET, Pabellón 1 Ciudad Universitaria, 1428EGA Buenos Aires, Argentina, ${ }^{2}$ MUNTREF Tecnópolis, Universidad Nacional de Tres de Febrero, Buenos Aires, Argentina.
\end{abstract}

\begin{abstract}
Reliable crossmodal correspondences between basic tastes and music features have been found in recent studies [1,2]. In this work, we explore associations between scales, chords and tastes. Several of these elementary musical structures show non-random patterns of matching with basic tastes. Moreover, their aggregate dyadic consonance [3] anti-correlates with the relative frequency of their matching to bitter taste.
\end{abstract}

\section{Online association experiment}

Forty five non-musicians (26 females; age 356 years) filled a Google Form survey. They were presented with 25 short sound files, each containing a scale or a chord, and asked to match a single taste word to each of them, among the four basic taste words bitter, sweet, salty and sour. All scales and chords were presented in a MIDI classical piano timbre, in the middle C4 octave. Audios were mastered to have a homogeneous loudness of where is the duration of the corresponding sound file. Each participant listened the audio files over earphones, adjusting the computer sound volume to a comfortable level with a practice video and then leaving it fixed. The scales and chords were based on $C$. The duration of chords and scales was fixed to $2 \mathrm{~s}$ and 0.5 s respectively; scales were presented in ascending and descending form. E

Scale types were: Major, Minor Melodic, Minor Harmonic, Chromatic, Whole-tone, Octatonic. Chord types were: Major triad, Minor triad, Diminished triad, Augmented triad, Minor 7th, Major 7th, Minor major 7th, Diminished 7th, Dominant 7th, Half-diminished 7th, Augmented 7th, Augmented major 7th, French augmented 6th.

\section{Results}

Associations for each taste are shown in Figure 1. We ran a chi-squared test under the null hypothesis of random responses ( $25 \%$ for each taste). For 13 out of the 25 musical structures the null hypothesis was rejected (significance level of 0.01).
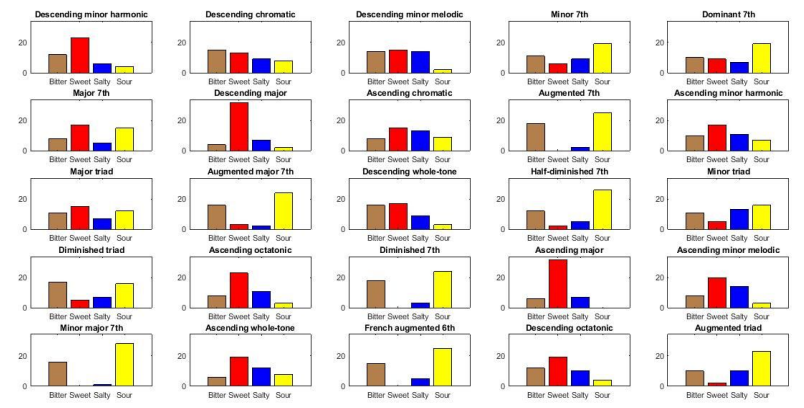

Figure 1 | Frequency of taste matches for each musical stimulus for the $\mathbf{4 5}$ participants.

These 13 structures are shown in Figure 2, where we performed a hierarchical clustering of the taste frequency profiles based on Euclidean distance. Two main clusters emerged: scales (mainly sweet or bitter and sweet associations) and chords (mainly sour or bitter and sour).

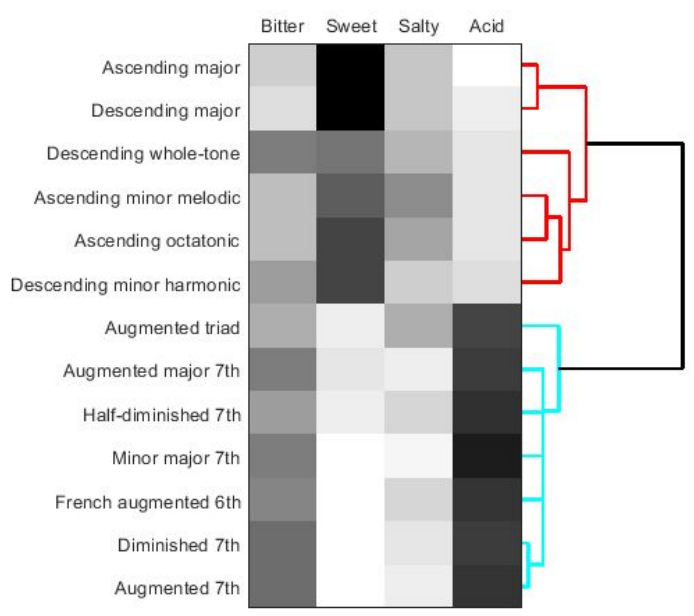

Figure 2 | Proportion of taste matches and clustering for the 13 structures with non-random matches.

\section{Aggregate Dyadic consonance and bitter taste}

To test the influence of interval content or melodic/ harmonic consonance of the scales and chords on taste choices, we computed aggregate dyadic consonance [3] 
for each structure with non-random taste matchings. This measure is based on the interval vector obtained from the collection of pitches in the structure, and consists of a weighted average of the interval vector, where the weights of each interval class derive from empirical studies of consonance perception.

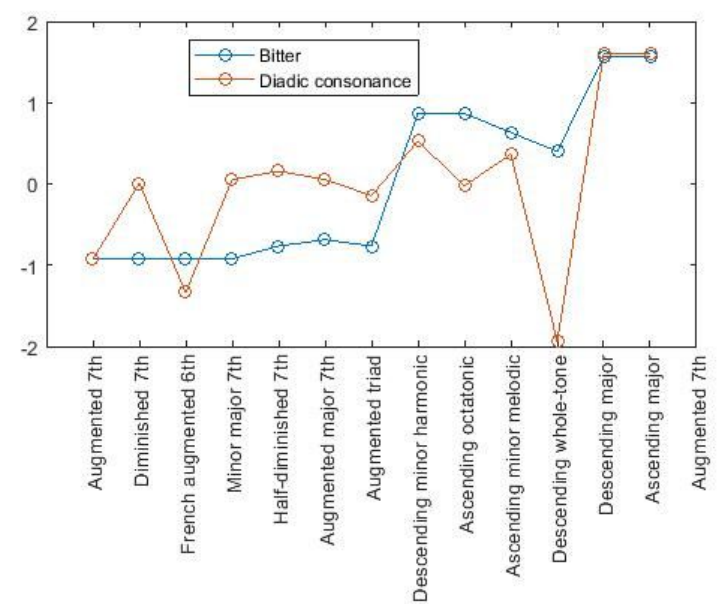

Figure 3 | Z-scores of Bitter matches and Aggregate Dyadic Consonance.

We computed Pearson correlation of this measure with frequency of responses for each taste, over all the 25 musical structures. There is a weak anticorrelation $(r=-0.4, p=0.046)$ in the case of bitter taste, and no significant correlation for the other tastes. The Z-scores of consonance and bitter responses are plotted in Figure 3.

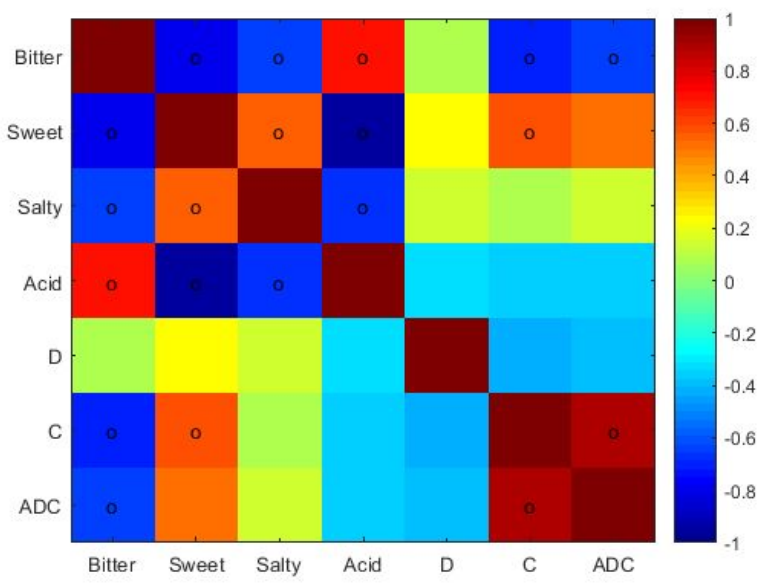

Figure 4 | Correlations between frequencies of taste matches and measures of consonance/dissonance: $D$ (Interval Dissonance Rate), C (Correlation consonance), ADR (Aggregate Dyadic Consonance). Circles indicate significant correlations (significance level of 0.05 ).

More generally, in Figure 4 we present correlations between the associated taste frequencies and three measures of consonance/dissonance: Interval
Dissonance Rate [4], Correlation consonance [3] and Aggregate dyadic consonance. In agreement with previous results [1-2], we find positive correlations between consonance and sweetness, and a strong anticorrelation between sour and sweet taste-music associations.

\section{Discussion}

Previous work on crossmodal correspondences has focused either on single notes or complex music. Here we considered the intermediate domain of basic music scales and chords, keeping constant the duration, intensity, register and timbre of the audio files. We show that the appearance of correspondences still occurs in this case, and that scales differ from chords in their matched taste profiles. In agreement with previous results [3], bitter and sweet present a negative and positive correlation respectively with consonance. However, dependence of sour on dissonance reported in other studies [2] were absent, suggesting that they may be context-dependent.

In the near future we plan to extend this study to consider a greater variety of musical structures and also include timbre as a variable parameter in the stimuli.

\section{References}

[1] Mesz, B., Trevisan, M. A., \& Sigman, M. (2011). The taste of music. Perception, 40(2), 209-219.

[2] Knöferle, K., \& Spence, C. (2012). Crossmodal correspondences between sounds and tastes. Psychonomic bulletin \& review, 1-15.

[3] Huron, D. (1994). Interval-class content in equally tempered pitch-class sets: Common scales exhibit optimum tonal consonance. Music Perception: An Interdisciplinary Journal, 11(3), 289-305.

[4] Mamedov, N., \& Peck, R. (2017). The Interval Dissonance Rate: An Analytical Look into Chromaticism of Chopin's Op. 10 No. 2 and Scriabin's Op. 11 No. 2. In Bridges 2017 Conference Proceedings (pp. 475-478). Tessellations Publishing. 